\title{
ESTUDO DA CINÉTICA DE ADSORÇÃO E MODELAGEM DO CORANTE VIOLETA CRISTAL EM ARGILA MARANHESE DE MONTES ALTOS
}

\author{
I. I. S. GUIDA ${ }^{1}$, S. S. FALCÃO ${ }^{1}$ \\ ${ }^{1}$ Universidade Federal Do Maranhão, Departamento de Engenharia Química \\ E-mail para contato: italoiury15@gmail.com
}

RESUMO - A contaminação de recursos hídricos pela indústria têxtil tem sido um grande problema ambiental. Entre as formas de tratamento dos efluentes despejados está a adsorção. As argilas são bons adsorventes de baixo custo e se mostraram como saída no tratamento dessas águas residuais. Este estudo busca avaliar a cinética de adsorção do corante violeta cristal (VC) pela argila de Montes Altos e os parâmetros das equações dos modelos a partir da modelagem, para consolidar um meio de tratamento eficaz de fonte renovável e barata aos efluentes despejados na natureza. Foram testados dois modelos: pseudo-primeira ordem e pseudosegunda ordem para descrever o comportamento da cinética de adsorção do corante (VC) pelos argila de Montes Altos. Aplicando a modelagem matemática, foi possível calcular os parâmetros que regem os modelos cinéticos, onde o (k) é a constante de transferência de massa para os modelos apresentados e o $\left(\mathrm{q}_{\mathrm{e}}\right)$ é a capacidade máxima de adsorção teórica no equilíbrio. $\mathrm{O}$ valor de $(\mathrm{k})$ foi de $0,05600\left(\mathrm{~min}^{-1}\right)$ para a cinética de pseudo-primeira ordem com o $\left(\mathrm{R}^{2}\right)=$ 0,735 e $0,00156\left(\mathrm{~g} \cdot \mathrm{mg}^{-1} \mathrm{~min}^{-1}\right)$ para a cinética de pseudo-segunda ordem com $\left(\mathrm{R}^{2}\right)=0,996$, o que caracteriza uma cinética de pseudo-segunda ordem para o $(\mathrm{VC})$. $\mathrm{O}$ valor de $\left(\mathrm{q}_{\mathrm{e}}\right)$ experimental foi de 25,01 (m.g. $\left.{ }^{-1}\right)$, podemos então confirmar o bom ajuste a cinética de pseudosegunda ordem onde ( $\mathrm{q}_{\mathrm{e}}$ ) calculado pelo modelo foi de 25,31 (m.g. $\left.{ }^{-1}\right)$. Por fim, o tempo de equilíbrio para a adsorção do (VC) foi de 60 (min).

\section{INTRODUÇÃO}

A poluição ambiental tem se mostrado como um sério problema enfrentado pela sociedade trazendo consigo altos riscos à saúde humana, prejudicando principalmente a fauna e a flora. Entre vários problemas que o homem causa a natureza, a contaminação de efluentes a partir do descarte de águas residuais de processos industriais é de grande preocupação para a análise dos impactos ambientais causados pela mão humana a natureza. Entre as indústrias que mais despejam esses efluentes, a alimentícia e a têxtil são as que mais causam impactos ambientais através de água contaminada principalmente de corantes como a anilina utilizada para confeite de bolos. Contudo, a indústria têxtil utiliza corantes para a confecção de tecidos que em sua composição apresentam metais pesados como mercúrio, cromo entre outros que são altamente prejudiciais a vida (Monash e Pugazhenthi, 2009).

Esses metais presentes nos corantes podem sofrer lixiviação contaminando não somente rios e lagos, mas uma das principais fontes de água potável no nosso país, os lençóis freáticos. Nesse contexto em que o homem vem causando a natureza, e com intuito de prevenir a 
contaminação desses reservatórios de água, a adsorção se mostra como uma boa alternativa. $\mathrm{O}$ corante Cristal Violeta (CV) é também conhecido como Violeta básico 3, é amplamente usado como corante roxo para têxteis tais como algodão e seda, e em tintas de impressão (Mittal et al., 2010). Existem vários processos de purificação dessas águas no qual podemos citar a extração por solventes, troca iônica, entre outros, porém, muitos desses métodos são de um custo elevado além de alguns gerarem resíduos, o que é outro problema ambiental. No entanto, a adsorção desses corantes utilizando as argilas é extremamente eficaz e de baixo custo, pois elas podem ser encontradas por toda parte e são de fácil extração.

A adsorção é um fenômeno fisico-químico que consiste na agregação de determinadas espécies, (adsorvato) na superfície de um material (adsorvente) (Dabrowski, 2001). Há grandes vantagens desse processo quando comparado a outros. Esse método permite que o adsorvente possa ser regenerado a partir do processo de desorção, para posteriormente ser reutilizado. Isto equivale a uma vantagem deste método em relação a outros meios, que geralmente ocasionam formação de produtos tóxicos (Atadashi et al., 2011). As argilas são poderosos adsorventes, pois em sua superficie ha uma grande concentração de cargas, o que favorece a adsorção, além de serem encontradas em todos os lugares do planeta (Lopes et al., 2014), porém, dependendo da classe de argila utilizada, é necessário um tratamento químico para melhor utilização de sua capacidade de adsorção já que na natureza as argilas encontram-se misturadas a outros compostos como a areia entre outros, o que não favorece a adsorção.

Esse estudo busca então avaliar a cinética de adsorção da argila maranhense de Montes Altos na remoção do corante violeta cristal e avaliar o melhor modelo que descreve a cinética de adsorção assim como os parâmetros das equações dos modelos a partir da modelagem matemática, para consolidar um meio de tratamento eficaz de fonte renovável e barata aos efluentes despejados na natureza.

\section{MATERIAIS E MÉTODO}

\subsection{Preparo do adsorvente e ativação química}

O adsorvente foi coletado na cidade de Montes Altos, cuja as coordenadas geográficas são, Latitude $(\mathrm{S})=05^{\circ} 48^{\prime} 26^{\prime}$ "e Longitude $(\mathrm{W})=47^{\circ} 10^{\prime} 08^{\prime \prime}$ utilizando todas as técnicas de amostragem de solos adequandas para obtenção da argila ha uma profundidade de aproximadamente $30 \mathrm{~cm}$ a parti do solo. Todos os reagentes utilizados são de ordem analítica, a solução do corante violeta cristal (VC) foi preparada a partir de uma solução estoque e diluída para a concentração de $100 \mathrm{mg} / \mathrm{L}$.

A argila passou por limpeza manual e sucessivas lavagens para retirada de resíduos e materiais inorgânicos e orgânicos. Logo após, foi seca em estufa à $60^{\circ} \mathrm{C}$ por $24 \mathrm{~h}$. Ainda in natura, o material foi triturado e peneirado para obtenção da granulometria $\mathrm{x}<75,0 \mu \mathrm{m}$ mais especificamente de $25 \mu \mathrm{m}$. A ativação química foi realizada com $1000 \mathrm{~g}$ da amostra anteriormente tratada. Para a ativação foi usada uma solução a 3,0 mol/L de $\mathrm{HCl}$ na proporção de 1 para 10. Preparou-se a solução de $\mathrm{HCl}$ 3,0 mol/L. Pesou-se $100 \mathrm{~g}$ da argila de Montes Altos em um beck e utilizou-se $1000 \mathrm{ml}$ da solução 3,0 mol/L de $\mathrm{HCl}$. Montou-se um sistema de agitação utilizando uma manta magnética, um balão de fundo chato, um recipiente adequado 
de alumínho contendo gliserina utilizada para o banho maria e controle da temperatura e um agitador magnético.

Utilzou-se também um condensador para que o ácido não vaporizasse. Após todo o sistema montado, adcionou-se o adosvente, a solução de $\mathrm{HCl}$ e a agitação foi ligada. A temperatura foi controlada utilizando um termômetro em $90^{\circ} \mathrm{C}$. O adsorvente ficou em contato com o $\mathrm{HCl}$ durante $2 \mathrm{~h}$. Após as duas horas, a solução contendo o adsorvente foi colocada em um banho com gelo para que a temperatura baixasse e depois todo o material foi lavado com água destilada utilizando um sistema de fitração a vácuo até a condutibilidade constante próxima a da água destilada. Foi medido também o pH e o teor de cloreto utilizando uma soução de nitrato de prata até não formar nenhum precipidado. Após a condutibilidade constante,o material foi levado para estufa a $60{ }^{\circ} \mathrm{C}$ e depois de seco foi macerado novamente e passado pelas peneiras de $0,088 \mathrm{~mm}$ obtentendo as partículas que se acumularam na peneira de $25 \mu \mathrm{m}$. O rendimento do material após todo o procedimento foi de $47 \%$ do total de $100 \mathrm{~g}, 47 \mathrm{~g}$ em unidade de massa.

\subsection{Cinética de adsorção e modelagem matemática}

Nos estudos cinéticos, 0,100 g do adsorvente foi colocado em contato com $25,0 \mathrm{~mL}$ da solução do corante com concentração de $100,0 \mathrm{mg} \mathrm{L}^{-1}$ e pH $=10$ nos tempos de 0 a 360,0 min, sob agitação de $150 \mathrm{rpm}$ a $25^{\circ} \mathrm{C}$. As cinéticas foram adequadas aos modelos de pseudo-primeira ordem e pseudo-segunda ordem conforme as Equações 1 e 2 já linearizadas (Lagergren, 1898) e (Ho et al., 1996).

$$
\log \left(q_{e}-q_{t}\right)=\log \left(q_{e}\right)-\frac{k}{2,303} t
$$

Onde, $k$ é a constante de taxa de adsorção de pseudo-primeira ordem $\left(\mathrm{min}^{-1}\right)$, $t$ é o tempo de reação $(\mathrm{min}), q_{e}$ é a capacidade de adsorção no equilíbrio $\left(\mathrm{mg} \mathrm{g}^{-1}\right)$ e $q_{\mathrm{t}}$ é a capacidade de adsorção em um tempo t (mg.g $\left.\mathrm{g}^{-1}\right)$.

$$
\frac{t}{q_{t}}=\frac{1}{k q_{e}^{2}}+\frac{1}{q_{e}} t
$$

Onde, $k$ é a constante de velocidade de adsorção de pseudo-segunda ordem $\left(\mathrm{g}, \mathrm{mg}^{-1} \cdot \mathrm{min}^{-}\right.$ $\left.{ }^{1}\right), q_{e}$ é a capacidade de adsorção no equilíbrio $\left(\mathrm{mg} \mathrm{g}^{-1}\right), q_{t}$ é a capacidade de adsorção em um tempo $\mathrm{t}\left(\mathrm{mg} \cdot \mathrm{g}^{-1}\right)$ e $t$ é o tempo de reação ( $\left.\mathrm{min}\right)$. Os ensaios foram realizados em batelada. A absorbância foi medida no comprimento de onda ( $\lambda$ ) $590 \mathrm{~nm}$ para o corante violeta cristal, por espectrofotometria UV-visível e a quantidade adsorvida foi dada pela Equação 3:

$$
q=\frac{\left(\mathrm{C}_{\mathrm{i}}-\mathrm{C}_{\mathrm{f}}\right) \times \mathrm{V}}{\mathrm{m}}
$$

Sendo $q$ a quantidade adsorvida $\left(\mathrm{mg} \mathrm{g}^{-1}\right)$; Ci e Cf são, respectivamente, as concentrações inicial e final dos corantes $\left(\mathrm{mg} \mathrm{L}^{-1}\right) ; \mathrm{m}(\mathrm{g})$ é a massa do adsorvente e $\mathrm{V}$ (L) é o volume de solução do corante utilizado. 


\section{RESULTADOS E DISCUSSÃO}

\subsection{Cinética de adsorção para o corante violeta cristal e modelagem matemática}

Com o intuito de conhecer algumas características sobre a cinética de adsorção do corante na argila, todos os dados experimentais obtidos no estudo cinético foram aplicados a dois modelos: pseudo-primeira ordem e pseudo-segunda ordem. Para a validação quantitativa, é necessário que os coeficientes de correlação sejam comparados. Pode-se verificar esse coeficiente a partir dos ajustes lineares de cada equação referente ao modelo cinético aplicado.

Considerando o mecanismo de adsorção, os modelos cinéticos foram ajustados aos dados experimentais, para o sistema, solução do corante violeta cristal e argila de Montes altos através da linearização das equações diferenciais que regem os modelos de pseudo-primeira ordem e pseudo-segunda ordem obtendo-se as Equações 1 e 2. Pode-se observar na Figura 1 que foram construídos os gráficos de $\log \left(q_{\max }-q_{t}\right)$ vs $t$ para o modelo de pseudo-primeira ordem e $t / q_{t}$ vs $t$ para o modelo de pseudo-segunda ordem respectivamente.

Figura 1 - Modelos cinéticos de pseudo-primeira ordem (1 - a), e pseudo-segunda ordem (1 -

b) ajustados a cinética de adsorção do corante violeta cristal em argila de Montes altos.
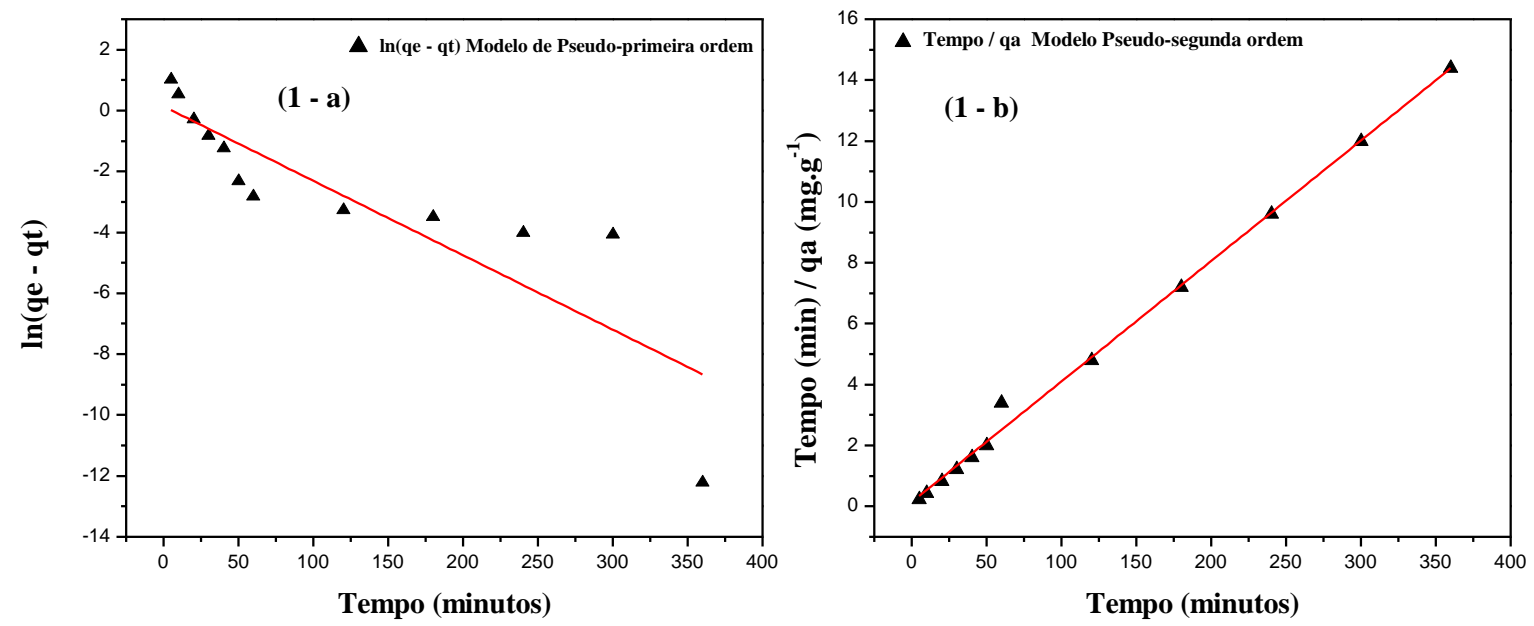

Analisando a Figura 1, percebe-se que a figura $(1-$ a) para o modelo de pseudoprimeira ordem, os dados cinéticos não se ajustaram bem. Já para a Figura $(1-$ b), o modelo cinético de pseudo-segunda ordem teve melhor ajuste. A Tabela 1 traz os dados obtidos a partir dos ajustes lineares para os modelos cinéticos apresentados.

Tabela 1 - Dados dos ajustes lineares a partir dos gráficos para os modelos cinéticos de pseudo-primeira ordem e pseudo-segunda ordem para o corante violeta cristal

\begin{tabular}{|c|c|c|c|c|}
\hline $\begin{array}{c}\text { Concentração da solução } \\
(\mathbf{m g . g})\end{array}$ & Modelos cinéticos & $\begin{array}{c}\mathbf{q e} \\
(\mathbf{m g . g})\end{array}$ & $\mathbf{k}$ & $\mathbf{R}^{\mathbf{2}}$ \\
\hline \multirow{2}{*}{100} & Pseudo-primeira ordem & 0,97 & $\begin{array}{c}0,05600\left(\mathrm{~min}^{-1}\right. \\
\left.{ }^{-1}\right)\end{array}$ & 0,735 \\
\cline { 2 - 5 } & Pseudo-segunda ordem & 25,31 & $\begin{array}{c}0,00156 \\
\left(\mathrm{~g} \cdot \mathrm{mg}^{-1} \mathrm{~min}^{-1}\right)\end{array}$ & 0,996 \\
\hline
\end{tabular}


A partir da análise dos dados na Tabela 1, percebe-se que o modelo de pseudo-segunda ordem se ajustou melhor aos dados, já que seu coeficiente de ajuste $\left(\mathrm{R}^{2}\right)$ foi de 0,996 , diferente do modelo de pseudo-primeira ordem, onde o $\left(R^{2}\right)$ foi de 0,735 . Com os coeficientes angular e linear da reta obtida pelo modelo de pseudo-segunda ordem linearizado, foi possível calcular a capacidade de adsorção teórica $q_{e}$ que foi de 25,31 (mg.g $\left.\mathrm{g}^{-1}\right)$. Comparando a capacidade de adsorção no equilíbrio teórica com a experimental que foi de aproximadamente $25,01\left(\mathrm{mg} \cdot \mathrm{g}^{-1}\right)$, percebe-se que o modelo descreve bem a cinética de adsorção do sistema solução do corante (VC) e argila de Montes Altos.

A Figura 2 traz a cinética de adsorção do sistema VC e argila de montes altos. Observa-se que o tempo de equilíbrio nestas condições foi de aproximadamente $60 \mathrm{~min}$ para o (VC). As quantidades adsorvidas aumentaram com o acréscimo do tempo de contato favorecendo a difusão do corante no meio até atingir o equilíbrio elevando a eficiência de adsorção das espécies em função da energia térmica do meio (Souza, 2009).

Figura 2 - Cinética de adsorção do corante violeta cristal pela argila de Montes altos.

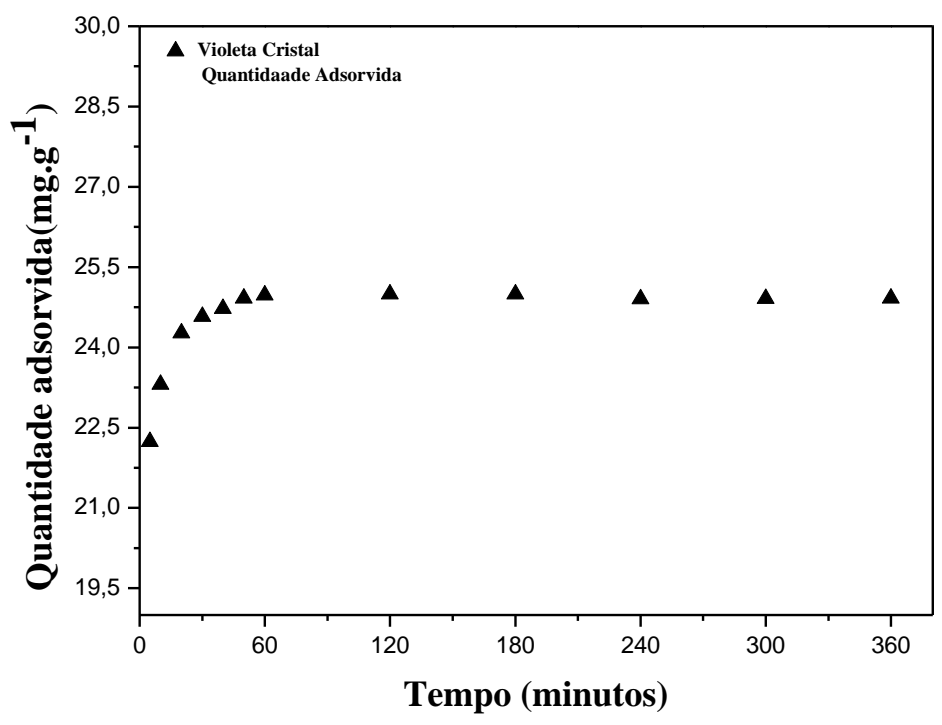

Ainda com base na Figura 2 em que o tempo de equilíbrio foi estimado em 60 minutos, percebe-se que a capacidade de adsorção foi diminuindo gradativamente até que depois de algum tempo o material já não adsorvia mais nada mesmo que o tempo passasse de $360 \mathrm{mim}$. Isso se deu devido à superfície do adsorvente está praticamente preenchida pelo corante e assim os sítios ativos do adsorvente já não interagiam mais com o corante.

\section{CONCLUSÃO}

Analisando os resultados obtidos a partir do estudo cinético do corante violeta cristal utilizando a argila de Montes Altos, podemos concluir que o modelo cinético de pseudosegunda ordem se ajustou bem aos dados. A cinética de adsorção nos deu o tempo de equilíbrio do adsorvente, sendo que após esse tempo, (60 mim), o material não adsorve mais nada, sendo assim aproximado ao modelo cinético de pseudo-segunda ordem. 
Ainda em relação a adsorção, a argila maranhense de Montes Altos se mostrou como um bom adsorvente na remoção de corantes catiônicos com uma quantidade adsorvida $(q)$ de 25,31 (mg.g ${ }^{-1}$ ), quando comparada a adsorventes padrão como o carvão ativado onde (Araki, 2013) encontrou um $(q)$ de aproximadamente $18\left(\mathrm{mg} \mathrm{g}^{-1}\right)$ em carvão ativado de bagaço de canade-açúcar, apesar da quantidade adsorvida não ter variado muito, e o equilíbrio tenha sido atingido em aproximadamente 1 hora para a argila maranhense.

Portanto, os objetivos desse estudo foram satisfeitos, já que as argilas são abundantes em todo o planeta terra, de baixo custo e se mostram como poderosos adsorventes no tratamento de efluentes provenientes da indústria têxtil e alimentícia.

\section{REFERÊNCIAS}

ATADASHI, I. M. Refining technologies for the purification of crude biodiesel. Applied Energy. v. 88, p. 4239-4251, 2011.

ARAKI, P. H. H. Adsorção de corante azul reativo 222 em carvão ativado produzido a partir do bagaço da cana-deaçúcar. Trabalho de conclusão de curso - Universidade Tecnológica Federal do Paraná, Apucarana, 2013.

DABROWSKI, A. Adsorption - from theory to practice. Advances in Colloid and Interface Science, v. 93, n. 1-3, p. 135-224, 2001.

HO, Y. S, WASE, D. A. J., FORSTER, C. F. Kinetic studies of competitive heavy metal adsorption by sphagnum moss peat. Environmental Technology. 17, 71-77, 1996.

LAGERGREN, S. On the theory of so-called adsorption dissolved substances. Handlingar Band. 24, 1-39, 1898.

LOPES, T. J. Adsorption of anthocyanins using clay-polyethylene nanocomposite particles. Applied Clay Science, v. 87, n. 0, p. 298-302, 2014.

MONASH, P.;PUGAZHENTHI, G. Adsorption of crystal violet dye from aqueous solution using mesoporous materials synthesized at room temperature. Adsorption, v. 15, p. 390405, 2009.

MITTAL, A., MITTAL, J., MALVIYA, A., KAUR, D., GUPTA, V. K. Adsorption of hazardous dye crystal violet from wastewater by waste materials. Journal of Colloid and Interface Science. 343, 463-473, 2010.

SOUZA, J. L. Mesocarpo do coco verde (Cocos nucifera) como adsorvente para corantes: turquesa remazol e azul remazol. Dissertação (Mestrado em Química) - Universidade Federal do Maranhão ,São Luis , 2009. 